\title{
Laparoscopic diaphragmatic plication for diaphragmatic paralysis and eventration: An objective evaluation of short-term and midterm results
}

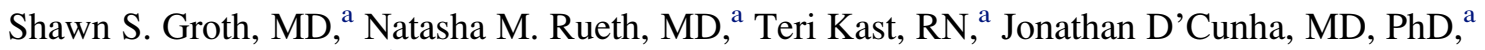 \\ Rosemary F. Kelly, MD, ${ }^{\mathrm{b}}$ Michael A. Maddaus, MD, FACS, ${ }^{\mathrm{a}}$ and Rafael S. Andrade, MD ${ }^{\mathrm{a}}$
}

\begin{abstract}
Objectives: We sought to objectively assess our outcomes after laparoscopic diaphragmatic plication for symptomatic hemidiaphragmatic paralysis or eventration using a respiratory quality-of-life questionnaire and pulmonary function tests.
\end{abstract}

\begin{abstract}
Methods: We performed a retrospective review of all symptomatic patients with hemidiaphragmatic paralysis or eventration who underwent laparoscopic diaphragmatic plication from March 1, 2005, through August 31, 2008. Patients with primary neuromuscular disorders were excluded from our analysis. We collected St George's Respiratory Questionnaire scores (a respiratory quality-of-life questionnaire) and pulmonary function test results preoperatively and at 1 month and 1 year postoperatively. A 2-sided significance level of .05 was used for all statistical testing.
\end{abstract}

Results: During the study period, 25 patients underwent laparoscopic diaphragmatic plication ( 9 right-sided and 16 left-sided plications); 1 patient required conversion to a thoracotomy. St George's Respiratory Questionnaire total scores $(59.3 \pm 26.8)$ improved by more than 20 points on average (a reduction of $\geq 4$ points after an intervention is considered a clinically significant improvement). This improvement was statistically significant at 1 month $(36.6 \pm 15.9, P=.001)$ and maintained significance at 1 year $(30.8 \pm 18.8, P=.009)$. Similarly, percent predicted maximum forced inspiratory flow $(93.2 \% \pm 34.1 \%)$ was significantly improved 1 month after plication $(113.9 \% \pm 31.8 \%, P=.01)$ and maintained significance at 1 year $(111.5 \% \pm 30.9 \%, P=.02)$.

Conclusions: Our objective evaluation of laparoscopic diaphragmatic plication for hemidiaphragmatic paralysis or eventration demonstrated significant short-term and midterm improvements in respiratory quality of life and pulmonary function test results. This approach represents a potential paradigm shift in the surgical management of hemidiaphragmatic paralysis or eventration. (J Thorac Cardiovasc Surg 2010;139:1452-6)

Diaphragmatic paralysis is an acquired condition resulting from spinal cord or phrenic nerve injury. ${ }^{1}$ Diaphragmatic eventration, on the other hand, is a rare congenital defect (incidence of $<0.05 \%$ ) in the muscular portion of the diaphragm. $^{2,3}$ Either condition leads to diaphragmatic elevation on radiologic examination; a radiologic finding without accompanying symptoms does not warrant surgical treatment. However, patients with symptomatic hemidiaphragmatic paralysis or eventration (ie, dyspnea, orthopnea, or both) benefit from hemidiaphragmatic plication to reduce dysfunctional cephalad diaphragmatic excursion during inspiration. ${ }^{4-6}$

\footnotetext{
From the Department of Surgery, Division of General Thoracic and Foregut Surgery, University of Minnesota, and the Department of Surgery, Division of Cardiothoracic Surgery, ${ }^{\mathrm{b}}$ Minneapolis Veterans Affairs Medical Center, Minneapolis, Minn. Disclosures: None.

Received for publication May 6, 2009; revisions received Aug 29, 2009; accepted for publication Oct 7, 2009; available ahead of print Jan 18, 2010

Address for reprints: Rafael S. Andrade, MD, Division of Thoracic and Foregut Surgery, MMC 207, 420 Delaware St SE, Minneapolis, MN 55455 (E-mail: andr0119@umn.edu).

0022-5223/\$36.00

Copyright (C) 2010 by The American Association for Thoracic Surgery doi:10.1016/j.jtcvs.2009.10.020
}

Traditionally, open and minimally invasive transthoracic diaphragmatic plications have been the approaches of choice for symptomatic hemidiaphragmatic paralysis or eventration. Recently, however, a laparoscopic approach to diaphragmatic plication was described by Huttl and colleagues. ${ }^{7}$ Laparoscopic diaphragmatic plication in 3 patients led to symptom resolution and significant improvements in pulmonary function test (PFT) results at a median follow-up of 72 months.

The aim of our study was to objectively assess our shortterm and midterm results of laparoscopic diaphragmatic plication for hemidiaphragmatic paralysis or eventration with the use of a respiratory quality-of-life questionnaire and PFTs.

\section{MATERIALS AND METHODS Data}

The Institutional Review Boards of the University of Minnesota and Minneapolis Veterans Affairs approved this retrospective review of our prospectively maintained database and waived the need to obtain informed consent for each patient. We studied the charts of all consecutive symptomatic patients 18 years of age and older with hemidiaphragmatic paralysis or eventration who underwent laparoscopic diaphragmatic plication at our institution from March 1, 2005 (the month we performed the first laparoscopic 


\section{Abbreviations and Acronyms \\ $\mathrm{FEV}_{1} \%=$ percent predicted forced expiratory volume in 1 second \\ FIFmax $\%=$ percent predicted maximum forced inspiratory flow \\ $\mathrm{FVC} \%=$ percent predicted forced vital capacity \\ PFT $=$ pulmonary function test \\ SGRQ $=$ St George's Respiratory Questionnaire}

diaphragmatic plication), through August 31, 2008. We collected information on patients' characteristics, perioperative details, chest radiographic findings, quality-of-life (St George's Respiratory Questionnaire [SGRQ]) scores, and PFT results. Patients with neuromuscular disorders were excluded from our analysis.

\section{Patient Evaluation}

We only offer a diaphragmatic plication to those patients with hemidiaphragmatic paralysis or eventration who are symptomatic (ie, dyspnea, orthopnea, or both) and have an elevated hemidiaphragm on a standard full-inspiration posteroanterior and lateral lateral chest X-ray. All patients who undergo evaluation for plication respond to the SGRQ to assess the effect of dyspnea on quality of life. Every patient undergoes PFTs to provide an additional objective parameter of response to plication; however, specific findings on PFTs do not guide the clinical decision to plicate. We obtain fluoroscopic sniff testing only if the cause of hemidiaphragmatic elevation is unclear; we do not perform a plication on patients with paralysis on sniff testing but no hemidiaphragmatic elevation on chest x-ray. Because the diagnosis of dyspnea or orthopnea caused by hemidiaphragmatic paralysis or eventration is predominately a diagnosis of exclusion, we thoroughly evaluate and attempt to correct other potential causes of dyspnea before deciding to plicate. For adults with phrenic nerve injury from cardiac surgery, we advocate a 12-month period of observation after cardiac surgery because phrenic nerve function can improve within that timeframe. ${ }^{8}$ We do not exclude patients on the basis of previous abdominal or thoracic surgery.

To assess the response to plication, we re-evaluate symptoms with the SGRQ, and we repeat PFTs.

SGRQ. We assessed quality of life with the SGRQ, a validated questionnaire that measures health impairment from respiratory disease and quantifies changes in health after therapy. ${ }^{9}$ The SGRQ has 2 parts. The first part, the symptoms score, assesses a patient's perception of the frequency and severity of their respiratory symptoms. The second part includes an activity score, which assesses the degree of impairment of daily physical activities by a respiratory disease, and an effect score, which assesses psychosocial dysfunction resulting from respiratory disease. The 2 parts are combined to produce a total score (range, 0-100; normal score, 6); higher scores indicate worse health impairment. After an intervention, a clinically significant change within or between groups is defined as a 4-point or greater reduction in SGRQ scores.

We collected SGRQ results at the following intervals: preoperatively, 1 month after plication, and 1 year after plication.

PFTs. We collected the following standard expiratory PFT results: percent predicted forced vital capacity $(\mathrm{FVC} \%)$ and percent predicted forced expiratory volume in 1 second $\left(\mathrm{FEV}_{1} \%\right)$. Because the diaphragm plays a critical role in inspiration, we also assessed an inspiratory PFT: percent predicted maximum forced inspiratory flow (FIFmax \%). All PFT results were collected in the upright position at the following intervals: preoperatively, 1 month after laparoscopic diaphragmatic plication, and 1 year after laparoscopic diaphragmatic plication. Although PFT results obtained in the supine position are expected to be at least $20 \%$ to $50 \%$ lower than in the upright position, we cannot routinely collect supine PFT results because many of our patients are referred from outside institutions and undergo follow-up studies at these facilities, where we cannot influence PFT acquisition practices. ${ }^{10-12}$

\section{Operative Technique}

All patients undergo general anesthesia with a single-lumen endotracheal tube. We use a 4-port technique (Figure 1). First, a 12-mm port (for a 10-mm $30^{\circ}$ camera) is placed $2 \mathrm{~cm}$ above the umbilicus and $2 \mathrm{~cm}$ lateral to the midline (toward the contralateral side of the affected hemidiaphragm). Two 12 $\mathrm{mm}$ (working) ports are placed in line with the first port, ipsilateral to the affected hemidiaphragm. A fourth 12-mm port (for the assistant) is placed near the xyphoid process.

After placing the ports and establishing pneumoperitoneum $(15 \mathrm{~mm} \mathrm{Hg})$, the affected hemidiaphragm is taut and displaced cephalad. We use electrocautery to create a 5-mm defect in the affected hemidiaphragm; the resultant pneumothorax causes the diaphragm to drop down, making it easy to grasp and retract caudally. Should respiratory or hemodynamic compromise occur, it can be easily corrected by placing a small $(<20 \mathrm{~F})$ chest tube. Because the opening in the diaphragm is small, the pneumoperitoneum is still maintained. Intrathoracic adhesions can be dissected by making a 5- to 6-cm diaphragm incision and accessing the thorax laparoscopically; the incision is then closed by incorporating it into the plication. A T-shaped plication is constructed with braided, nonabsorbable, number 2 (curved needle), hand-sewn, pledget-reinforced $\mathrm{U}$ stitches. We begin the plication by working in a posterior to anterior direction (starting as far posteriorly on the diaphragm as possible) and then proceeding from the medial to lateral direction (Figure 2). At the end of the operation, the plication is just tight enough that the addition of more plicating stitches would likely tear the diaphragm. We leave a chest tube in place until the output is less than $200 \mathrm{~mL} / \mathrm{d}$.

The procedure is carried out in a similar way on both sides, except for transection of the falciform ligament on the right side to allow the liver to drop slightly and to facilitate access to the posterior portion of the diaphragm.

\section{Statistical Analysis}

We used SAS version 9.1 (SAS Institute, Inc, Cary, NC) for data analysis. For all statistical testing, we used a 2-sided significance level of .05. Where appropriate, results are reported as means \pm standard deviations. To assess within-person changes before and after plication, we used

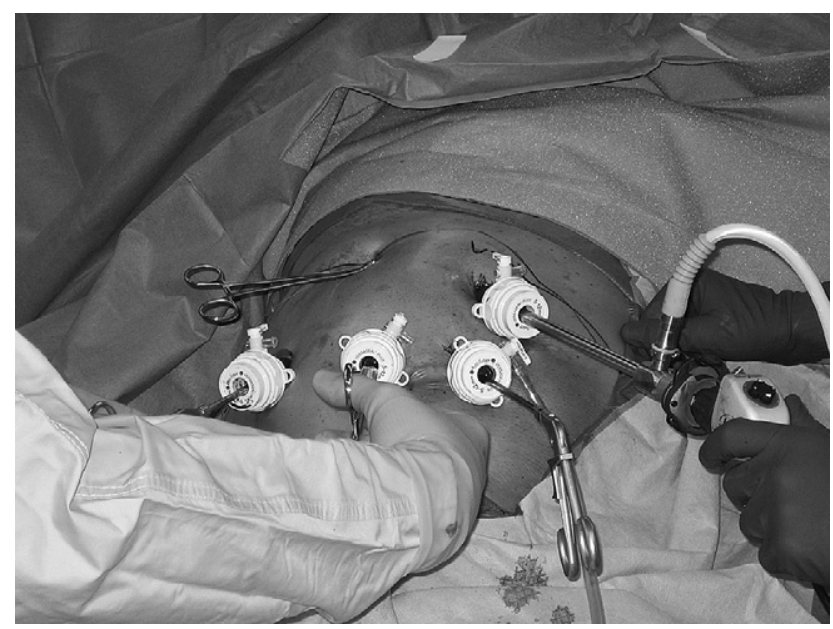

FIGURE 1. Port placement for laparoscopic plication of the right hemidiaphragm. 


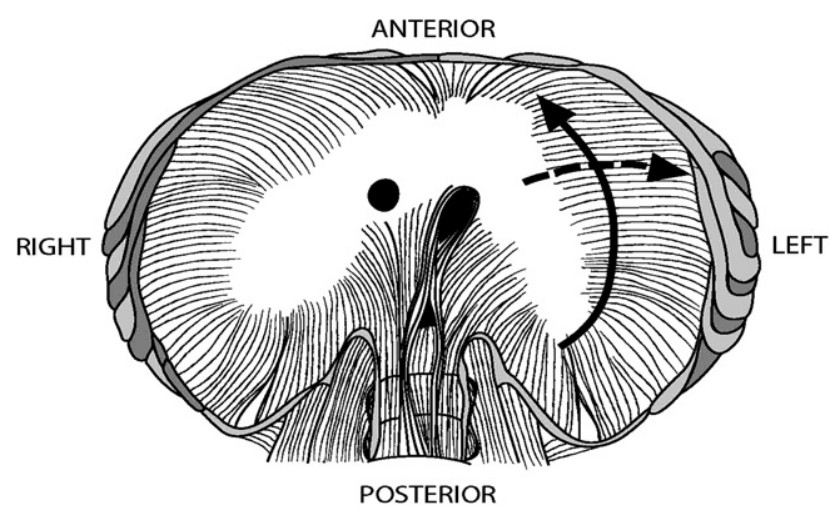

FIGURE 2. Diaphragmatic plication technique. Artwork by Lisa D'Cunha.

a matched-pairs $t$ test for parametric variables and a Wilcoxon signed-rank matched-pairs test for nonparametric variables.

\section{RESULTS}

Over the $3 \frac{1}{2}$-year study period, 25 patients with hemidiaphragmatic paralysis or eventration (mean age, $58.1 \pm$ 13.5 years; $64 \%$ men) underwent a laparoscopic diaphragmatic plication (9 right-sided and 16 left-sided plications). Two patients had a previous abdominal operation, 6 had a previous thoracic operation, and 3 had previous abdominal and thoracic operations. The median chest tube duration was 1 day (range, $0-20$ days). The median length of stay was 4 days (range, $1-11$ days).

After induction of pneumothorax, we did not have to abort the operation or convert to an open approach because of hemodynamic or respiratory compromise that did not resolve with chest tube placement when necessary. We had 1 conversion to thoracotomy caused by dense intrathoracic adhesions that could not be taken down laparoscopically or thoracoscopically. We encountered the following complica- tions in 5 patients ( $25 \%$ of our cohort): prolonged chest tube drainage ( $>200 \mathrm{~mL} / \mathrm{d}$ for $\geq 7$ days; $n=2$ ), pleural effusion requiring chest tube placement after discharge from the hospital $(\mathrm{n}=1)$, respiratory failure requiring reintubation $(\mathrm{n}=1)$, upper gastrointestinal hemorrhage $(\mathrm{n}=1)$, stroke $(\mathrm{n}=1)$, urinary tract infection $(\mathrm{n}=1)$, and paroxysmal atrial fibrillation $(n=1)$. There were no deaths.

One year after the operation, the plicated hemidiaphragm was lower than before the operation in all patients. Figure 3 displays a representative chest $\mathrm{X}$-ray film from one of our patients before, immediately after, and 1 year after plication.

Compared with preoperative scores, we found significant improvements in all components of the SGRQ at 1 month after laparoscopic diaphragmatic plication; this improvement was maintained at 1 year (Tables 1 and 2). Our patients experienced a 20-point or greater reduction in their scores at the 1-month and 1-year follow-up evaluations.

We also noted an improvement in their PFT results after laparoscopic diaphragmatic plication (Table 1). However, the improvement in FIFmax \% 1 year after plication did not reach statistical significance. One of our patients began smoking heavily again after the 1-month postoperative visit; his PFT results decreased at 1 year when compared with those at 1 month, but chest $\mathrm{x}$-ray showed a persistent plication effect. Given the negative effect of smoking on PFT results, we also analyzed the cohort's 1-year PFT data after excluding this patient's 1-year PFT results; we then found significant improvements in $\mathrm{FVC} \%, \mathrm{FEV} 1 \%$, and FIF$\max \%$ at 1 year after laparoscopic diaphragmatic plication (Table 2).

\section{DISCUSSION}

Our experience with laparoscopic diaphragmatic plication for symptomatic hemidiaphragmatic paralysis or eventration is the largest series reported to date. We demonstrated that

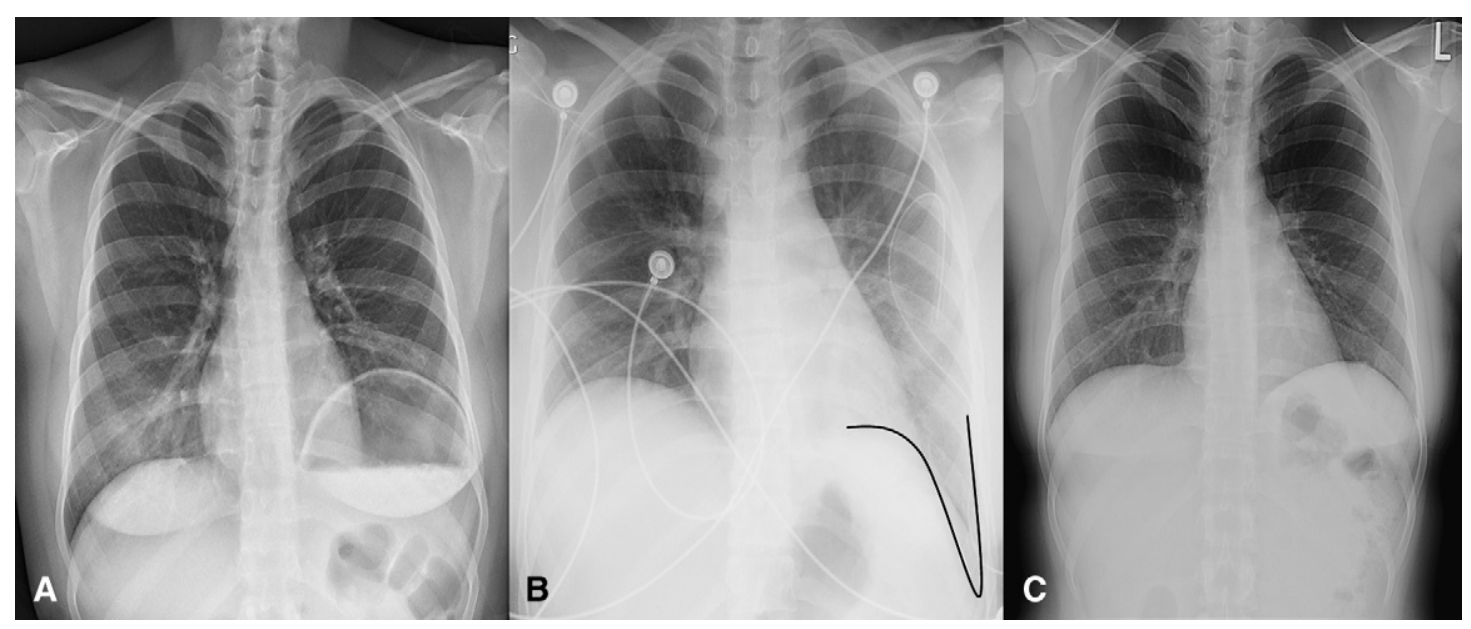

FIGURE 3. Representative chest $x$-ray film demonstrating a paralyzed left hemidiaphragm before plication (A) and immediately after plication (B; line added for emphasis). One year after plication, a normal chest $\mathrm{x}$-ray film is noted (C). 
TABLE 1. SGRQ and PFT results before and after laparoscopic diaphragmatic plication

\begin{tabular}{lccc}
\hline & & \multicolumn{2}{c}{ Postoperative } \\
\cline { 3 - 4 } & Preoperative & $\mathbf{1 ~ m o}$ & $\mathbf{1 ~ y}$ \\
\hline SGRQ scores & & & \\
Symptom score & $55.1 \pm 25.2$ & $34.5 \pm 19.5^{*}$ & $31.3 \pm 15.0^{*}$ \\
Activity score & $72.6 \pm 25.2$ & $58.6 \pm 21.8^{*}$ & $52.7 \pm 24.5^{*}$ \\
Impacts score & $49.3 \pm 30.7$ & $22.4 \pm 15.8^{*}$ & $25.7 \pm 12.2^{*}$ \\
$\quad$ Total score & $59.3 \pm 26.8$ & $36.6 \pm 15.9^{*}$ & $30.8 \pm 18.8^{*}$ \\
PFT & & & \\
FVC\% & $59.2 \pm 11.7$ & $65.3 \pm 9.4^{*}$ & $61.0 \pm 10.3^{*}$ \\
FEV $\%$ & $55.4 \pm 12.9$ & $62.5 \pm 11.9^{*}$ & $59.5 \pm 11.6^{*}$ \\
FIFmax $\%$ & $93.2 \pm 34.1$ & $113.9 \pm 31.8^{*}$ & $108.3 \pm 31.2$ \\
\hline
\end{tabular}

SGRQ, St George's Respiratory Questionnaire; $P F T$, pulmonary function test; $\mathrm{FVC} \%$, percent predicted forced vital capacity; $F E V_{l} \%$, percent predicted forced expiratory volume in 1 second; FIFmax $\%$, percent predicted maximum forced inspiratory flow. *Significantly different $(P<.05)$ from preoperative results.

laparoscopic diaphragmatic plication is safe, and we objectively assessed the benefits of laparoscopic diaphragmatic plication on symptoms and lung function.

\section{Functional Results}

The primary goal of diaphragmatic plication for hemidiaphragmatic paralysis or eventration is relief of dyspnea. Our patients experienced a dramatic 20-point reduction in SGRQ (respiratory quality-of-life) scores at the 1-month and 1-year follow-up evaluations (a successful intervention is one that leads to at least a 4-point reduction in SGRQ scores). ${ }^{9}$ The exact mechanisms responsible for improvement in respiratory symptoms after plication are uncertain. A combination of factors is likely responsible. Dysfunctional caudal excursion of a paralyzed or eventrated hemidiaphragm during inspiration results in impaired ventilation. In addition, perfusion to the basal portion of the lung ipsilateral to the paralyzed or eventrated diaphragm is impaired, possibly because of regional vasoconstriction induced by alveolar hypoxia. ${ }^{5}$ The resultant ventilation/perfusion mismatch and loss of pulmonary and chest wall compliance are among the factors that contribute to patients feeling dyspneic. By reducing diaphragmatic redundancy with a plication, the pathophysiologic factors that contribute to dyspnea (cranial displacement and dysfunctional caudal excursion during inspiration) are lessened, resulting in symptomatic improvement. ${ }^{4-6}$ In addition, experimental data show that the healthy (nonaffected) hemidiaphragm contracts more effectively after plication of the contralateral paralyzed hemidiaphragm. ${ }^{6}$

We also observed a significant and persistent improvement in PFT results after laparoscopic diaphragmatic plication. Not surprisingly, the absolute change in FIFmax \% appeared to be more evident than the absolute change in either $\mathrm{FEV}_{1} \%$ or $\mathrm{FVC} \%$ after laparoscopic plication. Because the diaphragm is an inspiratory muscle, we believe that in-
TABLE 2. SGRQ and PFT results before and after laparoscopic diaphragmatic plication after excluding data from patients who resumed smoking

\begin{tabular}{|c|c|c|c|}
\hline & \multirow[b]{2}{*}{ Preoperative } & \multicolumn{2}{|c|}{ Postoperative } \\
\hline & & $1 \mathrm{mo}$ & $1 \mathbf{y}$ \\
\hline \multicolumn{4}{|l|}{ SGRQ scores } \\
\hline Symptom score & $55.1 \pm 25.2$ & $34.5 \pm 19.5^{*}$ & $31.3 \pm 15.0^{*}$ \\
\hline Activity score & $72.6 \pm 25.2$ & $58.6 \pm 21.8^{*}$ & $52.7 \pm 24.5^{*}$ \\
\hline Impacts score & $49.3 \pm 30.7$ & $22.4 \pm 15.8^{*}$ & $25.7 \pm 12.2^{*}$ \\
\hline Total score & $59.3 \pm 26.8$ & $36.6 \pm 15.9^{*}$ & $30.8 \pm 18.8^{*}$ \\
\hline \multicolumn{4}{|l|}{ PFT } \\
\hline $\mathrm{FVC} \%$ & $59.2 \pm 11.7$ & $65.3 \pm 9.4^{*}$ & $61.0 \pm 10.6^{*}$ \\
\hline $\mathrm{FEV}_{1} \%$ & $55.4 \pm 12.9$ & $62.5 \pm 11.9^{*}$ & $60.9 \pm 10.7^{*}$ \\
\hline FIFmax $\%$ & $93.2 \pm 34.1$ & $113.9 \pm 31.8^{*}$ & $111.5 \pm 30.9^{*}$ \\
\hline
\end{tabular}

SGRQ, St George's Respiratory Questionnaire; $P F T$, pulmonary function test; $\mathrm{FVC} \%$, percent predicted forced vital capacity; $F E V_{l} \%$, percent predicted forced expiratory volume in 1 second; FIFmax $\%$, percent predicted maximum forced inspiratory flow. *Significantly different $(P<.05)$ from preoperative results.

spiratory parameters (eg, FIFmax \%) might more accurately reflect an improvement in diaphragmatic function.

Our results are consistent with the results from open transthoracic and thoracoscopic plication series. Multiple single-institution studies have demonstrated significant improvements in symptoms and respiratory function after an open transthoracic plication. ${ }^{13-16}$ In one such study of $17 \mathrm{pa}$ tients with unilateral paralysis, Graham and colleagues ${ }^{17}$ demonstrated that open transthoracic plication led to significant improvements in dyspnea, orthopnea, arterial oxygen tension, and PFT results (FVC, total lung capacity, and functional residual capacity assessed in both the sitting and supine position). Five- to 10-year follow-up data were available for $6(35 \%)$ of their patients, demonstrating that durable improvements in dyspnea scores, arterial oxygen tension, and PFT results can be achieved with this approach.

Similarly, multiple single-institution studies have demonstrated that thoracoscopic plication is associated with significant improvements in dyspnea and PFT results in patients with hemidiaphragmatic paralysis or eventration. ${ }^{18-20}$ In a report by Freeman and coworkers, ${ }^{18} 25$ patients with unilateral diaphragmatic paralysis were selected for thoracoscopic plication; it was successfully performed in 22 (3 required conversion to thoracotomy). At the 6-month follow-up, thoracoscopic plication was associated with significant improvements in dyspnea scores and PFT results.

Although open transthoracic plication is a time-honored technique that has demonstrated consistent and significant improvements in symptomatology and PFT results in patients with hemidiaphragmatic paralysis or eventration, there are several disadvantages of this approach: (1) a thoracotomy is required and (2) single-lung ventilation is required. Thoracoscopic plication circumvents the need for a thoracotomy; however, single-lung ventilation is still required. Furthermore, the working space for a thoracoscopic plication is limited both by the rib cage and by the elevated diaphragm. 
Compared with open transthoracic and thoracoscopic plication techniques, laparoscopic diaphragmatic plication offers several distinct advantages: (1) avoidance of singlelung ventilation, (2) ample working space and visualization, (3) potentially less postoperative pain, and (4) reduced risk of visceral injury from blind placement of transdiaphragmatic stitches.

Our conversion rate $(<5 \%)$ is comparable with published conversion rates during thoracoscopic plication (about 5\% to $10 \%$ ), highlighting the technical feasibility of a laparoscopic approach. ${ }^{18} \mathrm{~A}$ few technical points are worth noting: (1) we prefer hand-sewn stitches to automatic laparoscopic stitching devices because a curved (ie, $36 \mathrm{~mm}$ or $\mathrm{SH}$ ) needle allows deeper and wider tissue bites; (2) we believe that pledgets add strength to the repair, particularly in a very thin diaphragm; and (3) intrathoracic adhesions can generally be dissected laparoscopically through a 5- to 7-cm opening in the diaphragm, and the opening is then closed by incorporating it into the plication. (On occasion, an intrathoracic port can be of assistance if the dissection is difficult.)

Finally, our morbidity and mortality rates are comparable with published outcomes after open transthoracic and thoracoscopic plications, highlighting the safety of the laparoscopic approach.

\section{Limitations}

We recognize several limitations of our study. Our study lacks a control group. We have no longitudinal data on patients with hemidiaphragmatic paralysis or eventration who do not undergo plication. In addition, we do not have objective outcome data for patients who undergo alternative approaches (ie, thoracoscopic plication) because laparoscopic plication is the preferred approach at our institution. Finally, the sample size of our cohort (25 patients) was relatively small, and we have limited follow-up data for analysis (up to 1 year).

\section{CONCLUSION}

In conclusion, we demonstrated that laparoscopic diaphragmatic plication leads to significant objective improvements in respiratory quality of life and pulmonary function at midterm follow-up in patients with hemidiaphragmatic paralysis or eventration. We believe that laparoscopic diaphragmatic plication circumvents the limitations of open transthoracic and thoracoscopic plication techniques and appears to be as effective at treating dyspnea in patients with hemidiaphragmatic paralysis or eventration.

\section{References}

1. Riley EA. Idiopathic diaphragmatic paralysis; a report of eight cases. Am J Med. 1962;32:404-16.

2. Deslauriers J. Eventration of the diaphragm. Chest Surg Clin North Am. 1998;8: 315-30.

3. Thomas TV. Congenital eventration of the diaphragm. Ann Thorac Surg. 1970; 10:180-92.

4. Gibson GJ. Diaphragmatic paresis: pathophysiology, clinical features, and investigation. Thorax. 1989;44:960-70.

5. Ridyard JB, Stewart RM. Regional lung function in unilateral diaphragmatic paralysis. Thorax. 1976;31:438-42.

6. Takeda S, Nakahara K, Fujii Y, Matsumura A, Minami M, Matsuda H. Effects of diaphragmatic plication on respiratory mechanics in dogs with unilateral and bilateral phrenic nerve paralyses. Chest. 1995;107:798-804.

7. Hüttl TP, Wichmann MW, Reichart B, Geiger TK, Schildberg FW, Meyer G. Laparoscopic diaphragmatic plication: long-term results of a novel surgical technique for postoperative phrenic nerve palsy. Surg Endosc. 2004;18:547-51.

8. Summerhill EM, El-Sameed YA, Glidden TJ, McCool FD. Monitoring recovery from diaphragm paralysis with ultrasound. Chest. 2008;133:737-43.

9. Jones PW, Quirk FH, Baveystock CM. The St George's Respiratory Questionnaire. Respir Med. 1991;85(suppl B):25-37.

10. Clague HW, Hall DR. Effect of posture on lung volume: airway closure and gas exchange in hemidiaphragmatic paralysis. Thorax. 1979;34:523-6.

11. Gould L, Kaplan S, McElhinney AJ, Stone DJ. A method for the production of hemidiaphragmatic paralysis. Its application to the study of lung function in normal man. Am Rev Respir Dis. 1967;96:812-4.

12. McCredie M, Lovejoy FW, Kaltreider NL. Pulmonary function in diaphragmatic paralysis. Thorax. 1962;17:213-7.

13. Wright CD, Williams JG, Ogilvie CM, Donnelly RJ. Results of diaphragmatic plication for unilateral diaphragmatic paralysis. J Thorac Cardiovasc Surg. 1985;90: 195-8.

14. Higgs SM, Hussain A, Jackson M, Donnelly RJ, Berrisford RG. Long term results of diaphragmatic plication for unilateral diaphragm paralysis. Eur J Cardiothorac Surg. 2002;21:294-7.

15. Ciccolella DE, Daly BD, Celli BR. Improved diaphragmatic function after surgical plication for unilateral diaphragmatic paralysis. Am Rev Respir Dis. 1992;146: $797-9$.

16. Versteegh MI, Braun J, Voigt PG, Bosman DB, Stolk J, Rabe KF, et al. Diaphragm plication in adult patients with diaphragm paralysis leads to long-term improvement of pulmonary function and level of dyspnea. Eur J Cardiothorac Surg. 2007;32:449-56.

17. Graham DR, Kaplan D, Evans CC, Hind CR, Donnelly RJ. Diaphragmatic plication for unilateral diaphragmatic paralysis: a 10-year experience. Ann Thorac Surg. 1990;49:248-52.

18. Freeman RK, Wozniak TC, Fitzgerald EB. Functional and physiologic results of video-assisted thoracoscopic diaphragm plication in adult patients with unilateral diaphragm paralysis. Ann Thorac Surg. 2006;81:1853-7.

19. Mouroux J, Venissac N, Leo F, Alifano M, Guillot F. Surgical treatment of diaphragmatic eventration using video-assisted thoracic surgery: a prospective study. Ann Thorac Surg. 2005;79:308-12.

20. Suzumura Y, Terada Y, Sonobe M, Nagasawa M, Shindo T, Kitano M. A case of unilateral diaphragmatic eventration treated by plication with thoracoscopic surgery. Chest. 1997;112:530-2. 